\title{
Impacto da terapia antirretroviral na depressão e transtorno do sono em pacientes portadores de HIV
}

\author{
Impact of antiretroviral therapy on depression and sleep disorders in HIV patients
}

Impacto de la terapia antirretroviral en la depresión y los trastornos del sueño en pacientes con

\section{Resumo}

Depressão e alteração do sono são frequentes em pacientes portadores de HIV. O objetivo deste artigo é detectar alterações no sono e depressão relacionados a terapia antirretroviral na população adulta com HIV/aids. Aplicadas as escalas: Patient Health Questionnaire-9 (PHQ-9), Escala de Sonolência Epworth, adaptada ao Brasil (ESS-Br), e do Questionário Índice de Qualidade do Sono de Pittsburgh (PSQI). CEP: 3.319.628. Na avaliação do sono (PSQI), uso de Dolutegravir (DTG) apresentou 58,8\% sono de qualidade ruim, Efavirenz (EFZ) foi 38\% indicativo de sono ruim e 28,5\% de distúrbio do sono, e Atazanavir/ritonavir (ATV-r) com sono de qualidade ruim encontrado em 40\%. A detecção de qualquer tipo de alteração de sono não diferiu estatisticamente entre uso de EFZ ou DTG (p = 0,79). Com relação à avaliação da sonolência ESS-Br, detectamos 4,7\%, 17,2\% e $20 \%$ alteração importante dos pacientes em uso de EFZ, DTG e ATV, respectivamente. A análise obtida quanto a depressão (PHQ-9) foi: moderadamente grave (EFZ $=10 \%, \mathrm{DTG}=20,3 \%, \mathrm{ATV}=0 \%)$ e grave $(\mathrm{EFZ}=15 \%, \mathrm{DTG}=6,2 \%, \mathrm{ATV}=0 \%)$. Este estudo mostrou que indivíduos HIV positivos sofrem de distúrbios do sono independentes do regime de TARV, mas com maior impacto com o Efavirenz. A sonolência diurna estava presente em um de cada 5 indivíduos em uso de TARV. E a maior gravidade da depressão foi achado importante para os grupos em uso de Efavirenz e Dolutegravir.

Palavras-chave: Distúrbio do sono; HIV; Depressão; Terapia antirretroviral; Efavirenz; Dolutegravir; Atazanavir.

\begin{abstract}
Depression and sleep disorders are frequent in patients with HIV. The aim of this article is to detect sleep disorders and depression related to antiretroviral therapy in the adult population with HIV/AIDS. The following scales were applied: Patient Health Questionnaire-9 (PHQ-9), Epworth Sleepiness Scale, adapted to Brazil (ESS-Br), and the Pittsburgh Sleep Quality Index Questionnaire (PSQI). CEP: 3.319.628. In the sleep assessment (PSQI), use of Dolutegravir (DTG) showed $58.8 \%$ poor sleep quality, Efavirenz (EFZ) was $38 \%$ indicative of poor sleep and $28.5 \%$ of sleep disorder, and Atazanavir-ritonavir (ATV-r) with poor quality sleep was found in $40 \%$. The detection of any type of sleep disorder did not differ statistically between the use of EFZ or DTG $(\mathrm{p}=0.79)$. Regarding the assessment of ESS-Br sleepiness, we detected $4.7 \%, 17.2 \%$ and $20 \%$ important alterations in patients using EFZ, DTG and ATV, respectively. The analysis obtained for depression (PHQ-9) was moderately severe $(\mathrm{EFZ}=10 \%, \mathrm{DTG}=20.3 \%, \mathrm{ATV}=0 \%)$ and severe $(\mathrm{EFZ}=$ $15 \%$, DTG $=6.2 \%$, ATV $=0 \%$ ). This study showed that HIV-positive individuals suffer from sleep disturbances independent of the ART regimen, but with greater impact with Efavirenz. Daytime sleepiness was present in one in
\end{abstract}


every 5 individuals using ART. And the greater severity of depression was found to be important for the groups using Efavirenz and Dolutegravir.

Keywords: Sleep disorders; HIV; Depression; Antiretroviral therapy; Efavirenz; Dolutegravir; Atazanavir.

\begin{abstract}
Resumen
La depresión y los trastornos del sueño son frecuentes en pacientes con VIH. El objetivo de este artículo es detectar los trastornos del sueño y la depresión relacionados con la terapia antirretroviral en la población adulta con VIH / SIDA. Se aplicaron las siguientes escalas: Patient Health Questionnaire-9 (PHQ-9), Epworth Sleepiness Scale, adaptada a Brasil (ESS-Br) y Pittsburgh Sleep Quality Index Questionnaire (PSQI). CEP: 3.319.628. En la evaluación del sueño (PSQI), el uso de Dolutegravir (DTG) mostró un 58.8\% de mala calidad del sueño, el Efavirenz (EFZ) fue un 38\% indicativo de sueño deficiente y un $28.5 \%$ de trastorno del sueño, y el Atazanavir-ritonavir (ATV-r) con sueño de mala calidad se encontró en el 40\%. La detección de cualquier tipo de trastorno del sueño no difirió estadísticamente entre el uso de EFZ o DTG $(\mathrm{p}=0,79)$. En cuanto a la valoración de la somnolencia ESS-Br, detectamos alteraciones importantes del 4,7\%, 17,2\% y 20\% en pacientes que utilizan EFZ, DTG y ATV, respectivamente. El análisis obtenido para la depresión (PHQ-9) fue: moderadamente grave $(\mathrm{EFZ}=10 \%, \mathrm{DTG}=20,3 \%, \mathrm{ATV}=0 \%)$ y grave $(\mathrm{EFZ}=15 \%, \mathrm{DTG}=$ $6,2 \%, \mathrm{ATV}=0 \%)$. Este estudio mostró que las personas VIH positivas sufren alteraciones del sueño independientes del régimen de TAR, pero con mayor impacto con Efavirenz. La somnolencia diurna estuvo presente en uno de cada 5 individuos que usaban TAR. Y se descubrió que la mayor gravedad de la depresión era importante para los grupos que usaban efavirenz y dolutegravir.
\end{abstract}

Palabras clave: Alteración del sueño; VIH; Depresión; Terapia antirretroviral; Efavirenz; Dolutegravir; Atazanavir.

\title{
1. Introdução
}

A infecção causada pelo vírus da imunodeficiência humana tipo 1 (HIV-1) continua sendo um problema de saúde pública mundial. De acordo com dados da Organização Mundial da Saúde (OMS), até o final de 2020, havia aproximadamente 37,7 milhões de pessoas infectadas pelo HIV em todo o mundo (WHO, 2021). Uma parcela significativa desta população pode vir a apresentar comprometimento cognitivo e psiquiátrico, mesmo em indivíduos que estão fazendo uso regular da terapia antirretroviral (TARV) (Troncoso \& Conterno, 2015; Chang et al., 2018; Nightingale et al., 2014).

O HIV compromete o sistema nervoso central (SNC) em praticamente todos os infectados, podendo permanecer latente por muitos anos. Dessa forma, estão-lhe associados múltiplos danos neuropsiquiátricos (Alves et al., 2013). A introdução da TARV possibilitou maior controle efetivo da replicação viral e melhora do estado imunológico, além de ter diminuído a incidência da maioria das doenças neurológicas oportunistas em pacientes infectados pelo HIV (Brew \& Chan, 2014; Haziot et al., 2015). No entanto, as alterações neurocognitivas associadas ao HIV (HAND) permanecem com prevalência significativa, variando de $15 \%$ a 50\%, mesmo em indivíduos em uso da TARV (BRASIL, 2018). Ao longo dos anos, o perfil clínico do comprometimento neurocognitivo mudou drasticamente, caracterizando-se por diminuição dos casos de HAD, caindo de $20 \%$ para menos de 5\% (Brew \& Chan, 2014). Em contrapartida, devido o SNC ser um santuário imunológico, ocorre uma maior incidência das formas leves e moderadas, asymptomatic neurocognitive impairment (ANI) e Mild neurocognitive disease (MND), a despeito dos avanços farmacológicos (Azevedo et al., 2014).

Além disso, a infecção pelo HIV tem um grande impacto na vida pessoal, afetiva, sexual, ocupacional e social dos indivíduos, gerando prejuízos na estruturação da sua rede de suporte social. Dada a sua associação a um curso maligno e implacável, os infectados são, muitas vezes, vítimas de estigma e discriminação (Alves et al., 2013). Em verdade, a prevalência de perturbações psiquiátricas associadas à infecção pelo HIV encontra-se entre $12 \%$ a 66\%, sendo inclusive mais frequentes que na população geral (Troncoso \& Conterno, 2015). O uso de alguns antirretrovirais também pode impactar como evento adverso neuropsiquiátrico, variando em frequência e gravidade (Zareifopoulos et al., 2020).

Nesse contexto, dentre os transtornos psiquiátricos mais comumente observados em pessoas que vivem com HIV (PVHIV), a ansiedade e a depressão são os mais prevalentes. Dentre eles, a depressão é o transtorno mais frequente, tendo estimativas de prevalência de 30\% a 61\%. Estima-se que as PVHIV tenham entre duas a sete vezes mais probabilidade de desenvolver depressão que a população geral, embora as taxas sejam díspares entre os estudos (Azevedo et al., 2014; Alves et 
al., 2013). No Brasil, a prevalência de depressão em PVHIV varia entre 32\% a 34,5\% (Troncoso \& Conterno, 2015). Vale ressaltar que, muitas vezes, o diagnóstico de depressão representa um desafio, uma vez que as sequelas da infecção, as doenças oportunistas e os efeitos colaterais do tratamento tendem a imitar sintomas de depressão como fadiga, perda ponderal, somatização, falta de apetite, alteração da concentração, entre outros (Kamat et al., 2015).

Adultos que vivem com uma doença crônica correm maior risco de problemas de sono e, dependendo da definição, problemas de sono são vivenciados por 30\% a 100\% dos adultos soropositivos. Não está claro que tipo de problema de sono em adultos com HIV experiência, ou se a sua perturbação do sono está relacionada com a própria infecção ou com o tratamento médico e os efeitos colaterais dos medicamentos. A perturbação do sono não é apenas prevalente, mas também um dos sintomas mais intensos e angustiantes experimentados nesta população. Na infecção por HIV, sono insatisfatório tem sido associado à progressão da doença, terapia medicamentosa, situação de emprego e falta de conhecimento sobre comportamentos que promovem um bom sono. O tipo específico de distúrbio do sono experimentado por esta população precisa ser mais bem compreendido a fim de fornecer a intervenção mais eficaz para melhorar os sintomas, funcionamento diurno e qualidade de vida (Lee et al., 2012).

Uma grande parcela dos pacientes em uso de TARV no Brasil e no mundo utiliza o esquema que combina Tenofovir, Lamivudina e Efavirenz. Após estudos randomizados comprovarem a segurança do Dolutegravir, um inibidor de integrase, e demonstrarem até mesmo sua superioridade em relação ao Efavirenz, o Sistema Único de Saúde (SUS) brasileiro passou a ofertar gratuitamente esse novo antirretroviral a partir do ano de 2017 (VITORIA et al, 2018). Apesar de muitos estudos sobre a epidemiologia e as covariáveis dos distúrbios do sono em adultos infectados pelo HIV, pouco se sabe sobre os tipos específicos de problemas de sono que ocorrem nessa população. Portanto, o objetivo deste artigo é descrever o tempo total de sono, bem como os problemas para adormecer e permanecer dormindo na população adulta com HIV/AIDS, aplicando conhecidos instrumentos para investigação de alterações neuropsiquiátricas (Depressão e Distúrbios do Sono) e correlacionar com terapia antirretroviral em pacientes com HIV acompanhados em ambulatório especializado.

\section{Metodologia}

\subsection{Desenho do Estudo}

Tratou-se de um estudo transversal, descritivo, cujos dados foram obtidos por meio de revisão de prontuário e autoaplicação de três escalas que avaliam depressão e transtorno do sono. Parecer de aprovação pelo CEP local com numeração CAAE: 10790919.1.0000.5049 e número do parecer: 3.319.628.

\subsection{Local de Estudo}

O estudo foi realizado em Serviço de Atendimento Especializado (SAE) de pacientes com HIV/aids que está localizado dentro da Clínica Escola do Centro Universitário Unichristus, sendo vinculado ao município de Fortaleza. O mesmo conta com atendimento multidisciplinar e farmácia para dispensação exclusiva de antirretrovirais. Durante atendimento farmacêutico individualizado foi oferecido ao paciente os questionários e aplicados após assinatura do Termo de Consentimento Livre e Esclarecido, logo após sendo avaliado por médico infectologista do SAE. O período de recrutamento e aplicação de questionários foi de março a junho de 2019. Os dados epidemiológicos do paciente foram coletados dos prontuários de julho a outubro de 2019.

\subsection{Participantes}

A amostra foi constituída por pacientes que realizam seguimento ambulatorial na Clínica Escola de Saúde de um centro universitário da cidade de Fortaleza - CE. Os pacientes foram selecionados por livre demanda na farmácia da clínica. O SAE 
acompanha cerca de 600 pacientes regularmente, sendo a amostra selecionada equivalente a $15 \%$ do total.

Critérios de inclusão: Pacientes maiores de 18 anos, portadores de infecção pelo HIV, atendidos no ambulatório de Infectologia de uma clínica escola de saúde; Pacientes alfabetizados, capazes de responder individualmente os questionários.

Critérios de exclusão: Pacientes que se recusaram a participar da pesquisa; Pacientes com dados incompletos ou que preencheram as escalas e/ou o questionário de maneira inadequada.

\subsection{Variáveis e Escalas aplicadas}

Foram aplicadas as escalas: Patient Health Questionnaire-9 (PHQ-9), Escala de Sonolência Epworth, adaptada ao Brasil (ESS-Br) e, Questionário Índice de Qualidade do Sono de Pittsburgh (PSQI).

O PHQ-9 constitui-se de nove perguntas que avaliam a presença de cada um dos sintomas para o episódio de depressão maior, descritos no Manual Diagnóstico e Estatístico dos Transtornos Mentais (DSM-IV) 13. Os nove sintomas consistem em humor deprimido, anedonia (perda de interesse ou prazer em fazer as coisas), problemas com o sono, cansaço ou falta de energia, mudança no apetite ou peso, sentimento de culpa ou inutilidade, problemas de concentração, sentir-se lento ou inquieto e pensamentos suicidas. Esta versão está disponível online http://www.phqscreeners.com, acessado em 12/Set/2012 (Bertolazi at al. 2011). A frequência de cada sintoma nas últimas duas semanas é avaliada em uma escala Likert de 0 (nenhuma vez), 1 (vários dias), 2 (mais da metade dos dias) e 3 (quase todos os dias). O questionário inclui uma pergunta que avalia a interferência desses sintomas no desempenho de atividades diárias, como trabalhar e estudar (Santos et al., 2013).

A escala de Sonolência de Epworth é uma ferramenta utilizada para aferir o grau de sonolência diurno. Ela foi desenvolvida pelo Dr.Murray Johns e considera aspectos do dia a dia e modo de vida recente. De acordo com os resultados, ela pode sugerir a possibilidade de o paciente estar sofrendo de algum distúrbio do sono como a hipersonia diurna, apneia obstrutiva do sono e insônia. Durante a avaliação é necessário responder um questionário com notas que vão de 0 a 3 que representam as chances de cochilar em diferentes ações do dia a dia. Quanto maior a pontuação, maiores são os indícios de algum tipo de distúrbio do sono (Empana et al., 2009).

O PSQI avalia a qualidade e perturbações do sono durante o período de um mês e foi desenvolvido por Buysse et al., sendo um questionário padronizado, simples e bem aceito pelos pacientes. O instrumento é constituído por 19 questões em autorelato e cinco questões direcionadas ao cônjuge ou acompanhante de quarto. As últimas cinco questões são utilizadas apenas para a prática clínica, não contribuindo para a pontuação total do índice. As 19 questões são categorizadas em sete componentes, graduados em escores de: zero (nenhuma dificuldade) a três (dificuldade grave).

Os componentes do PSQI são:

- C1 qualidade subjetiva do sono,

- C2 latência do sono,

- C3 duração do sono,

- C4 eficiência habitual do sono,

- C5 alterações do sono,

- C6 uso de medicamentos para dormir

- C7 disfunção diurna do sono.

A soma dos valores atribuídos aos sete componentes varia de zero a vinte e um no escore total do questionário, indicando que quanto maior o número pior é a qualidade do sono. Um escore total maior que cinco indica que o indivíduo está apresentando grandes disfunções em pelo menos dois componentes, ou disfunção moderada em pelo menos três componentes, sendo considerada como sono ruim (Araújo et al., 2015). 


\subsection{Análise de dados e Estatística}

Os dados foram inseridos dentro de uma planilha de Excel, onde foram trabalhados os dados epidemiológicos de frequência. Os resultados de cálculo e somatória do PSQI foi realizado por definição de fórmula no próprio Excel. Foram excluídos da análise final os questionários que não foram finalizados completamente. Os dados epidemiológicos foram extraídos diretamente do documento fonte que é o prontuário do paciente. A análise estatística foi feita utilizando o programa GraphPrisma (https://www.graphpad.com), sendo os valores de p considerados estatisticamente significativo <0,05, com intervalo de confiança de 95\%. Aplicados testes de Qui quadrado de Pearson e Fisher, de acordo com a numeração obtida pelos dados, sendo assim analisadas as comparações entre os diferentes esquemas antirretrovirais (ATV-r, EFZ e DTG).

\section{Resultados e Discussão}

O presente estudo analisou dados de 92 participantes, sendo 86,95\% homens ( $\mathrm{N}=80)$, com média da idade do total de participante de 36,1 anos. Quanto ao esquema antirretroviral em uso 71,73\% estava com DTG (N=66), seguido de $22,82 \%$ com EFZ (N=21) e 5,43\% em uso de ATV-r ( $=5)$. No entanto, após avaliar o preenchimento adequado dos questionários, a amostra total final foi comporta de: 89 participantes nas avaliações das escalas PSQI e PHQ-9 e, 88 na escala ESS-Br.

Com base na avaliação da qualidade do sono, dos pacientes em uso de terapia com DTG 58,8\% (N=37) apresentaram PSQI maior ou igual a 5, que é interpretado como sono de qualidade ruim, e 11,2\% (N=7) apresentaram escore maior que 10 que equivale a presença de distúrbio de sono. Em relação ao EFV 38\% (N=8) deste grupo denotaram PSQI indicativo de sono ruim e 28,5\% (N=6) de distúrbio do sono. Na análise do grupo em uso de ATV-r evidenciou-se sono de qualidade ruim em 40\% (N=2) da amostra e com distúrbio do sono também em $40 \%(\mathrm{~N}=2)$, sendo importante ressaltar a pequena quantidade de pacientes nesse grupo, o que pode representar um viés de avaliação para esse antirretroviral (Tabela 1).

Tabela 1 - Relação entre o esquema antirretroviral e a avaliação da qualidade de sono obtida pela escala PSQI.

\begin{tabular}{lllll}
\hline Esquema ARV & Boa & Ruim & Distúrbio do sono & $\begin{array}{l}\text { Total } \\
(\mathbf{N})\end{array}$ \\
\hline EFZ & $\mathbf{( 0 - 4 )}$ & $\mathbf{( 5 - 1 0 )}$ & $\mathbf{( > 1 0 )}$ & 21 \\
DTG & $7(33 \%)$ & $8(38 \%)$ & $6(28,5 \%)$ & 63 \\
ATV-r & $19(30 \%)$ & $37(58,8 \%)$ & $7(11,2 \%)$ & 5 \\
\hline
\end{tabular}

* ARV=antirretroviral, EFZ = Efavirenz, DTG = Dolutegravir, ATV-r = Atazanavir 300mg associado a ritonavir de 100mg. Fonte: Autores.

Em análise de todos os pacientes que tiveram qualquer tipo de alteração de sono $(69,6 \%)$, desde sono ruim a presença de distúrbio do sono, não havendo diferença estatística entre uso de EFZ ou DTG (p = 0,79), (Figura 1). As médias de pontos no estudo para cada um dos componentes da escala de PSQI foi comparada entre os três grupos de antirretrovirais, sendo para C1 maior para EFZ e DTG que representa como o paciente qualifica subjetivamente seu sono, equivalente entre os grupos para o componente C2 de latência ou tempo para iniciar o sono, menor para DTG no componente duração de sono que se relaciona a tempo de sono, menor eficiência habitual de sono para ATV-r que representa C4, distúrbios do sono que é C5 equivalente para EFZ e DTG mas maior para ATV-r, no componente C6 que se refere a utilização de medicamento para indução de sono 
observamos maior média para EFZ e ATV-r, e maior disfunção durante o dia com uso de ATV-r e equivalente entre EFZ e DTG que compõem o C7 (Tabela 2).

Tabela 2 - Média de pontos de cada um dos componentes da escala de PSQI com diferentes medicamentos antirretrovirais.

\begin{tabular}{llll}
\hline Componente do PSQI & EFZ & DTG & ATV \\
\hline Qualidade subjetiva do sono (C1) & 1,3 & 1,1 & 1,0 \\
Latência do sono (C2) & 1,3 & 1,2 & 1,2 \\
Duração do sono (C3) & 1,2 & 0,9 & 1,2 \\
Eficiência habitual do sono (C4) & 0,6 & 0,5 & 0,2 \\
Distúrbios do sono (C5) & 1,3 & 1,3 & 2,2 \\
Uso de medicação para dormir (C6) & 1,1 & 0,8 & 1,2 \\
Disfunção durante o dia (C7) & 1,0 & 0,9 & 1,4 \\
\hline
\end{tabular}

$\mathrm{EFZ}=$ Efavirenz, DTG $=$ Dolutegravir, ATV-r = Atazanavir 300mg associado a ritonavir de $100 \mathrm{mg}$. Fonte: Autores.

Figura 1 - Pontuação dos componentes da escala de PSQI com EFZ, DTG e ATV-r.

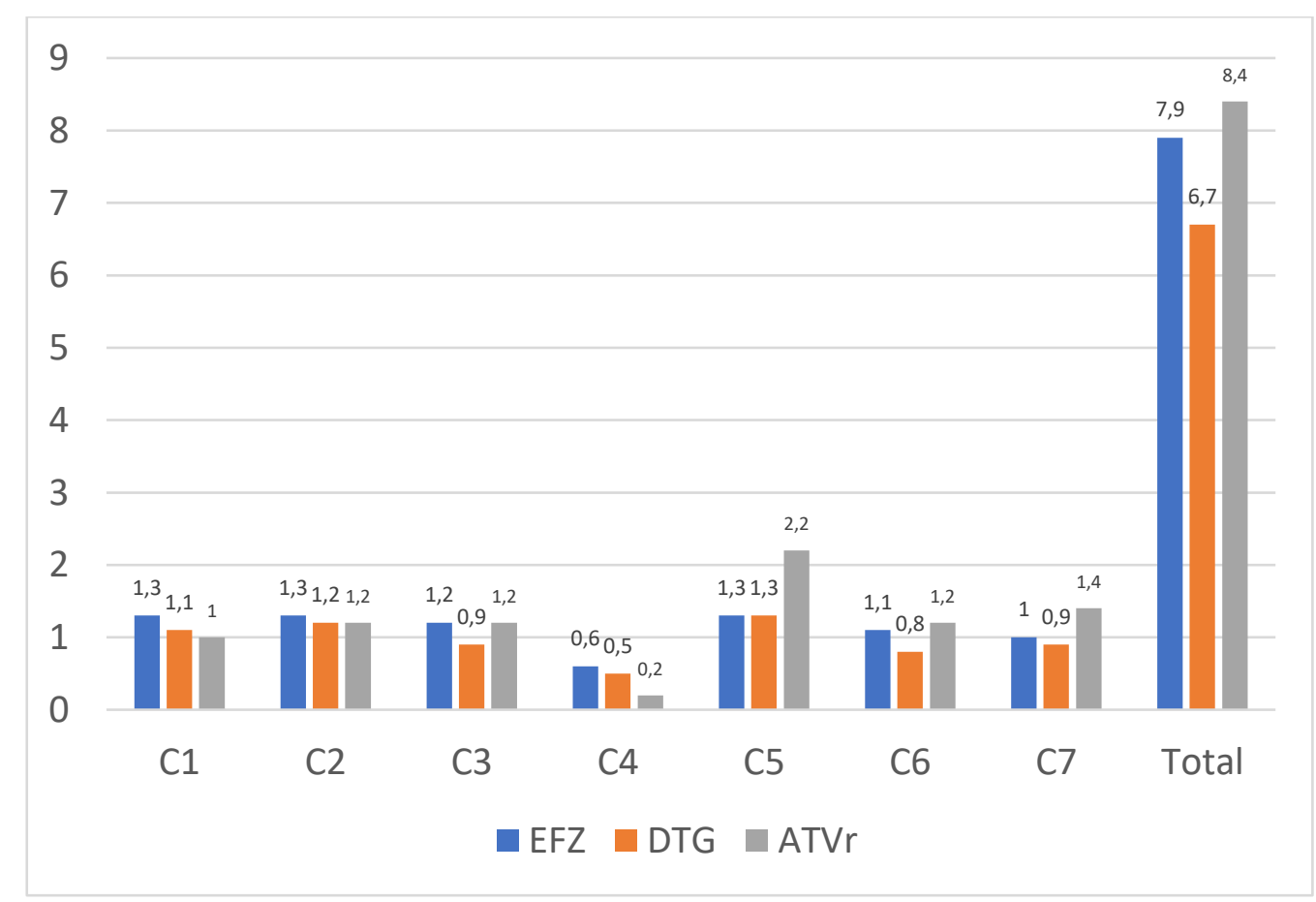

* C-C7 componentes da escala PSQI com médias de pontos para cada medicação. Na vertical pontuação da escala de 0-10. $\mathrm{ARV}=$ antirretroviral, $\mathrm{EFZ}=$ Efavirenz, DTG $=$ Dolutegravir, ATV-r $=$ Atazanavir 300mg associado a ritonavir de 100mg. Fonte: Autores.

Com relação à avaliação da sonolência diurna, através da ESS-Br, detectou-se escore maior que 10, caracterizado como presença de sonolência diurna excessiva (SDE) ou sonolência grave (SG) em 19,3\% da amostra. Sendo a SG mais frequente com uso de EFZ e a SDE com DTG (Tabela 3). 
Tabela 3 - Relação entre o esquema antirretroviral e a avaliação da SDE pela escala de ESS.

\begin{tabular}{|c|c|c|c|c|c|}
\hline Esquema ARV & $\begin{array}{l}\text { Normal } \\
(<11)\end{array}$ & $\begin{array}{l}\text { Sonolência Diurna } \\
\text { Excessiva (11-15) }\end{array}$ & $\begin{array}{l}\text { Sonolência } \\
\text { (>15) }\end{array}$ & grave & $\begin{array}{l}\text { Total } \\
(\mathbf{N})\end{array}$ \\
\hline EFZ & $16(76,2 \%)$ & $1(4,7 \%)$ & $2(9,5 \%)$ & & 19 \\
\hline DTG & $51(79,7 \%)$ & $11(17,2 \%)$ & $2(3,1 \%)$ & & 64 \\
\hline ATV & $4(80 \%)$ & $1(20 \%)$ & 0 & & 5 \\
\hline
\end{tabular}

$\mathrm{ARV}=$ antirretroviral, EFZ = Efavirenz, DTG = Dolutegravir, ATV-r $=$ Atazanavir 300mg associado a ritonavir de 100mg. Fonte: Autores.

A análise obtida quanto ao grau de severidade de depressão, através da escala PHQ-9, evidenciou que com uso de ATVr a maioria apresentava grau leve mesmo com amostragem pequena, e grau moderado e grave maior com uso de EFZ. Escores de depressão moderadamente grave e grave foram obtidos em 25\% dos pacientes em uso de EFZ e em 26,5\% dos pacientes em uso de DTG, sem diferença estatística $(\mathrm{p}=1)$, embora a maioria apresentasse maior prevalência de moderadamente grave com uso de DTG e grave com EFZ (Tabela 4).

Tabela 4 - Relação entre esquema antirretroviral e grau de severidade de depressão obtido pela escala de PHQ-9.

\begin{tabular}{lllllll}
\hline Esquema & Nenhuma & Leve & Moderada & Moderadamente & Grave & Total \\
ARV & $\mathbf{( 0 - 4 )}$ & $\mathbf{( 5 - 9 )}$ & $\mathbf{( 1 0 - 1 4 )}$ & grave (15-19) & $(\mathbf{2 0 - 2 7 )}$ & $\mathbf{( N )}$ \\
\hline EFZ & $8(40 \%)$ & $5(25 \%)$ & $2(10 \%)$ & $2(10 \%)$ & $3(15 \%)$ & 20 \\
DTG & $26(40,6 \%)$ & $16(25 \%)$ & $5(7,8 \%)$ & $13(20,3 \%)$ & $4(6,25 \%)$ & 64 \\
ATV & 0 & $4(80 \%)$ & $1(20 \%)$ & 0 & 0 & 5 \\
\hline
\end{tabular}

$\mathrm{ARV}=$ antirretroviral, $\mathrm{EFZ}=$ Efavirenz, DTG $=$ Dolutegravir, ATV-r $=$ Atazanavir 300mg associado a ritonavir de 100mg. Fonte: Autores.

Sobre a interferência psíquica do uso de TARV em pessoas vivendo com HIV (PVH), nosso estudo revelou taxas semelhantes e sem disparidades estatísticas de depressão moderadamente grave e grave entre pacientes em uso de EFV ou de DTG (23,8\% no EFV e 26,5\% no DTG). Esse dado assemelha-se às taxas da literatura, com prevalência de depressão em HIV de $12 \%$ na população entre 15 e 49 anos, que utilizou o instrumento de pesquisa PHQ-9, correspondendo a 16\% da população pré-TARV e a $9 \%$ da população em uso de TARV, no entanto não referente a um esquema antiviral específico. Além disso, ideação suicida esteve presente em $16 \%$ da amostra total, referente a $20 \%$ pré-TARV e a $11 \%$ da amostra em uso de TARV (Malava et al., 2018).

Ao abordar alterações psíquicas relacionadas especificamente ao uso de dolutegravir (DTG), consideramos os achados de 5 ensaios clínicos randomizados, sendo três duplo cegos, além da coorte do Observational Pharmaco-Epidemiology Research \& Analysis (OPERA, Hsu et al., 2018), que selecionou os sintomas psiquiátricos insônia, ansiedade, depressão e suicídio em pacientes em uso de DTG. Na análise dos conhecidos estudos SPRING-2 (Fettiplace et al., 2017), FLAMINGO (Molina et al., 2015), SINGLE (Walmsley et al., 2015), ARIA (Orrell et al., 2017) e SAILING (Cahn et al., 2013), os pacientes foram randomizados em grupos recebendo DTG ou outro comparador, sendo utilizados raltegravir (RAL) no SPRING-2 e no SAILING, EFV, tenofovir (TDF) e emtricitabina no SINGLE, darunavir com ritonavir (DRV-r) no FLAMINGO e atazanavir-ritonavir (ATV-r) no ARIA. Ao comparar os estudos, a referida pesquisa concluiu haver taxas baixas e semelhantes para cada um dos sintomas nos 5 estudos, com exceção para insônia que foi maior no estudo SINGLE, sendo referida em $17 \%$ dos pacientes em uso de DTG (71/417) e em 12\% daqueles em uso de EFV (52/419). Acerca da coorte OPERA, os sintomas de ansiedade, depressão ou insônia estiveram mais presentes em pacientes em uso de DTG e foram mais baixos naqueles em uso de EFV. 
Apesar disso, a interrupção do tratamento devido a estes sintomas foi mais baixa entre aqueles que usavam DTG. Ademais, a prevalência dos sintomas foi semelhante nos quatro medicamentos principal, exceto no RAL em que as taxas de ansiedade e depressão foram maiores. Ao finalizar a análise comparativa, concluiu-se que a frequência de sintomas psiquiátricos associados ao DTG era, em geral, baixa e semelhante a outros medicamentos combinados como terceira droga utilizados (Fettiplace et al., 2017).

Ademais, resultados contrastantes com o presente estudo e com outros dados da literatura foram vistos em recente trabalho multicêntrico, de caráter longitudinal, que reuniu dados coletados entre 1995 e 2017, acerca de 3.434 mulheres portadoras de infecção por HIV. A análise desses dados associou o uso de Efavirenz (29\%) à presença de sintomas depressivos, incluindo sintomas somáticos como alterações do sono. No entanto, a mesma associação não foi vista em mulheres que faziam uso de Dolutegravir (9\%), contrastando com a hipótese inicial do estudo e com outros dados presentes em literatura. Como possíveis justificativas para esse achado, sugeriu-se que o Dolutegravir não foi analisado especificamente, mas sim coletivamente com outros medicamentos antirretrovirais, além de possível pequeno tamanho da amostra estudada (Williams et al., 2020).

Um recente estudo de coorte avaliou o impacto no desempenho do sono e a presença de distúrbios neuropsiquiátricos após switch de uma terapia baseada em EFV para uma não-baseada em EFV. Na pesquisa, 32 indivíduos do sexo masculino em uso do esquema EFV, emtricitabina e tenofovir (EFV/FTC/TDF), sem fatores de risco tradicionais para apneia obstrutiva do sono (AOS), foram randomizados, 2:1, por 12 semanas: 10 continuaram a terapia com EFV e 22 realizaram switch para elvitegravir/cobicistat/emtricitabine/tenofovir (EVG/COBI/FTC/TDF). Realizaram-se, no início e ao fim de 12 semanas, polissonografia durante a noite e avaliações neuropsicológicas e do sono. Escores de Epworth iguais ou maiores a 11 foram observados em $3(9,4 \%)$ indivíduos e escores de PSQI de 5 ou mais foram observados em 59,4\% no início do estudo. Altas taxas basais de anormalidades do sono, avaliadas através do Índice de apneia ou hipopneia (IAH) e do Índice de Distúrbios Respiratórios (IDR), foram observadas nos pacientes em uso de terapia baseada em EFV. No entanto, escores de ESS e de PSQI não se correlacionaram com quaisquer parâmetros da arquitetura do sono. Não foram observadas diferenças significativas ao longo das 12 semanas entre os dois grupos em quaisquer parâmetros dos testes de sono ou neuropsicológico. Além disso, observou-se que anormalidades neuropsicológicas e do sono não revertem facilmente após descontinuação do EFV (Shikuma et al., 2018).

Os achados do nosso estudo corroboram com o evidenciado em literatura, onde os PVH apresentam uma alta prevalência de transtornos de sono e depressão, principalmente com a utilização de Efavirenz (Gutierrez et al., 2019; Santos et al., 2021; Santos et al., 2020). Sendo nesta população encontrado maior utilização de medicamentos para indução do sono e que repercutem tanto na polifarmácia quanto no maior risco de interações medicamentosas. Embora com baixa evidência de sonolência durante o dia na amostragem, esse efeito precisa ser detectado a fim e evitar acidentes com desfecho grave para o paciente.

\section{Conclusão}

Este estudo mostrou que indivíduos HIV positivos sofrem de distúrbios do sono independentes do regime de TARV, mas com maior impacto com o Efavirenz. A sonolência diurna estava presente em um em cada 5 indivíduos em uso de TARV. E a maior gravidade da depressão foi achado importante para os grupos em uso de Efavirenz e Dolutegravir.

Ainda não se pode afirmar com clareza a correlação entre sintomas neuropsiquiátricos com terapias antirretrovirais específicas, visto a etiologia multifatorial na patogênese desses sintomas em PVHIV, como antecedentes de condições psiquiátricas, ativação imune devido ao HIV, toxicidade antirretroviral, estigma de viver com o HIV e variáveis do estilo de vida, como uso de drogas e álcool que pode ser maior em parte dessa população. Entretanto, a presente análise corrobora com achados da literatura e suscita a necessidade de novos estudos, com amostras maiores, que avaliem as diferenças entre drogas específicas. 


\section{Referências}

Alves, R., Baptista, A. M., Moutinho, A., Moutinho, F., \& Teixeira, J. (2013) VIH: aspectos neuropsiquiátricos da infecção e da terapêutica. Psilogos: Revista do Serviço de Psiquiatria do Hospital Fernando Fonseca, 11, 64-70.

Araujo, P. A. B., Sties, S. W., Wittkopf, P. G., Schmitt Netto, A., Gonzáles, A. I., Lima, D. P., Guimarães, S. N., Aranha, E. E., \& De Carvalho, A. A. T. (2015). Pittsburgh sleep quality index for use in cardiopulmonary and metabolic rehabilitation. Rev Bras Med Esporte 21 (6).

Azevedo, R. L. W., Silva, E. A. A., Dionísio, L. R., \& Saldanha, A. A. W. (2014) Trastornosafetivos/cognitivos associados à TARV e à qualidade de vida no contexto da Aids. Revista Interamericana de Psicologia/Interamerican Journal of Psychology (IJP), 48(2), $238-251$.

Bertolazi, A. N., Fagondes, S. C., Hoff, L. S., Dartora, E. G., Miozzo, I. C., de Barba, M. E., \& Barreto, S. S. (2011). Validation of the Brazilian Portuguese version of the Pittsburgh Sleep Quality Index. Sleep Med. 12(1):70-5. 10.1016/j.sleep.2010.04.020.

Brasil; ministério da saúde (ms); secretaria de vigilância em saúde. (2018) Protocolo clínico e diretrizes terapêuticas para manejo da infecção pelo HIV em adultos. http://www.aids.gov.br/pt-br/pub/2013/protocolo-clinico-e-diretrizes-terapeuticas-para-manejo-da-infeccao-pelo-hiv-em-adultos

Brew, B. J., Chan, P. (2014) Update on HIV dementia and HIV-associated neurocognitive disorders. Current neurology and neuroscience reports, $14(8)$, 468.

Cahn P, Pozniak A. L, Mingrone H, Shuldyakov A, Brites C, Andrade-Villanueva JF, Richmond G, Buendia C. B, Fourie J, Ramgopal M, Hagins D, Felizarta F, Madruga J, Reuter T, Newman T, Small C. B, Lombaard J, Grinsztejn B, Dorey D, Underwood M, Griffith S, \& Min S; extended SAILING Study Team (2013). Dolutegravir versus raltegravir in antiretroviral-experienced, integrase-inhibitor-naive adults with HIV: week 48 results from the randomised, doubleblind, non-inferiority SAILING study. Lancet. 382(9893):700-8.

Chang, J. L., Tsai, A. C., Musinguzi, N., Haberer, J. E., Boum, Y., Muzoora, C., Bwana, M., Martin, J. N., Hunt, P. W., Bangsberg, D. R., \& Siedner, M. J. (2018) Depression and Suicidal Ideation Among HIV-Infected Adults Receiving Efavirenz Versus Nevirapine in Uganda: A Prospective Cohort Study. Ann Intern Med. Aug 7;169(3):146-155.

Empana, J. P., Dauvilliers, Y., Dartigues, J. F., Ritchie, K., Gariepy, J., Jouven, X., Tzourio, C., Amouyel, P., Besset, A., \& Ducimetiere, P. (2009) Excessive daytime sleepiness is an independent risk indicator for cardiovascular mortality in community-dwelling elderly: the three city study. Stroke. 40(4):1219-24.

Fettiplace, A., Stainsby, C., Winston, A., Givens, N., Puccini, S., Vannappagari, V., Hsu, R., Fusco, J., Quercia, R., Aboud, M., \& Curtis, L. (2017). Psychiatric Symptoms in Patients Receiving Dolutegravir. Journal of acquired immune deficiency syndromes (1999), 74(4), 423-431.

Gutierrez, J., Tedaldi, E. M., Armon, C., Patel, V., Hart, R., \& Buchacz, K. (2019). Sleep disturbances in HIV-infected patients associated with depression and high risk of obstructive sleep apnea. SAGE open medicine, 7.

Haziot, M., Barbosa Junior, S. P., Vidal, J. E., de Oliveira, F., \& de Oliveira, A. (2015). Neuroimaging of HIV-associated neurocognitive disorders. Dementia \& neuropsychologia, 9(4), 380-384.

Hsu, R., Fusco, J., Henegar, C., Mounzer, K., Wohlfeiler, M., Vannappagari, V., Aboud, M., Curtis, L., \& Fusco, G. (2018). Psychiatric outcomes observed in patients living with HIV using six common core antiretrovirals in the Observational Pharmaco-Epidemiology Research and Analysis database (OPERA). Therapeutic advances in drug safety, 9(12), 675-686.

Huang, X., Li, H., Meyers, K., Xia, W., Meng, Z., Li, C., Bai, J., He, S., Cai, W., Huang, C., Liu, S., Wang, H., Ling, X., Ma,P., Tan, D., Wang, F., Ruan, L., Zhao, H., Wei, H., Liu, Y., Yu, J., Lu, H., Wang, M., Zhang, T., Chen, H. \& Wu, H (2017). Burden of sleep disturbances and associated risk factors: A crosssectional survey among HIV-infected persons on antiretroviral therapy across China. Sci Rep 7, 3657.

Kamat, R, Cattie, J. E., Marcotte, T. D., Woods, S. P., Franklin, D. R., Corkran, S. H., Ellis, R. J., Grant, I., \& Heaton, R. K. (2015) Incident major depressive episodes increase the severity and risk of apathy in HIV infection. J Affect Disord. 175:475-80.

Lee, K.A., Gay, C., Portillo, C.J., Coggins, T., Davis, H., Pullinger, C. R., \& Aouizerat, B. E. (2012) Types of sleep problems in adults living with HIV/AIDS. J Clin Sleep Med. 8(1):67-75.

Malava, J. K., Lancaster, K. E., Hosseinipour, M. C., Rosenberg, N. E., O'Donnell, J. K., Kauye, F., Mbirimtengerenji, N., Chaweza, T., Tweya, H., Phiri, S., Pence, B. W., \& Gaynes, B. N. (2018). Prevalence and correlates of probable depression diagnosis and suicidal ideation among patients receiving HIV care in Lilongwe, Malawi. Malawi medical journal: the journal of Medical Association of Malawi, 30(4), 236-242.

Molina J. M., Clotet B., Lunzen J. v., Lazzarin A., Cavassini M., Henry K., Kulagin V., Givens N., Oliveira C. F., Brennan C., on behalf of the FLAMINGO study team (2015). Once-daily dolutegravir versus darunavir plus ritonavir for treatment-naive adults with HIV-1 infection (FLAMINGO): 96 week results from a randomised, open-label, phase 3b study. The Lancet 2(4), E127-E136.

Nightingale, S., Winston, A., Letendre, S., Michael, B. D., McArthur, J. C., Khoo, S., \& Solomon, T. (2014) Controversies in HIV-associated neurocognitive disorders. The Lancet Neurology, 13(11), 1139-1151.

Orrell, C., Hagins, D. P., Belonosova, E., Porteiro, N., Walmsley, S., Falcó, V., Man, C. Y., Aylott, A., Buchanan, A. M., Wynne, B., Vavro, C., Aboud, M., Smith, K. Y., \& ARIA study team (2017). Fixed-dose combination dolutegravir, abacavir, and lamivudine versus ritonavir-boosted atazanavir plus tenofovir disoproxil fumarate and emtricitabine in previously untreated women with HIV-1 infection (ARIA): week 48 results from a randomised, open-label, noninferiority, phase $3 \mathrm{~b}$ study. The lancet. HIV, 4(12), e536-e546.

Santos, I. S., Tavares, B. F., Munhoz, T. N., de Almeida, L. S. P., da Silva, N. T. B., Tams, B. D., Patella, A. M., \& Matijasevich, A. (2013) Sensibilidade e especificidade do Patient Health Questionnaire-9 (PHQ-9) entre adultos da população geral. Cadernos de Saúde Pública, 29, $1533-1543$.

Santos, E. E. P., Ribeiro, A. C., Paula, C. C. \& Padoin, S. M. M. (2020). From the discovery of diagnosis to antiretroviral treatment: phenomenological study with young people living with HIV. Research, Society and Development, 9(7): 1-16, e441974254. 
Research, Society and Development, v. 10, n. 12, e25101219989, 2021

(CC BY 4.0) | ISSN 2525-3409 | DOI: http://dx.doi.org/10.33448/rsd-v10i12.19989

Santos, G. S., Melo, G. C. de, Duprat, I. P., Brandão, T. M., \& Silva, Y. T. da. (2021). Depressive symptoms in people living with the Human Immunodeficiency Virus. Research, Society and Development, 10(6), e14710615493.

Shikuma, C. M., Kohorn, L., Paul, R., Chow, D. C., Kallianpur, K. J., Walker, M., Souza, S., Gangcuangco, L., Nakamoto, B. K., Pien, F. D., Duerler, T., Castro, L., Nagamine, L., \& Soll, B. (2018). Sleep and neuropsychological performance in HIV+ subjects on efavirenz-based therapy and response to switch in therapy. HIV clinical trials, 19(4), 139-147.

Troncoso, F. T., \& Conterno, L. O. (2015). Prevalence of neurocognitive disorders and depression in a Brazilian HIV population. Revista da Sociedade Brasileira de Medicina Tropical, 48(4), 390-398.

Vitoria, M., Hill, A., Ford, N., Doherty, M., Clayden, P., Venter, F., Ripin, D., Flexner, C., \& Domanico, P. L. (2018) The transition to dolutegravir and other new antiretrovirals in low-income and middle-income countries: what are the issues? AIDS. Jul 31;32(12):1551-1561. 10.1097/QAD.0000000000001845.

Williams, D. W., Li, Y., Dastgheyb, R., Fitzgerald, K. C., Maki, P. M., Spence, A. B., Gustafson, D. R., Milam, J., Sharma, A., Adimora, A. A., Ofotokun, I., Fischl, M. A., Konkle-Parker, D., Weber, K. M., Xu, Y., \& Rubin, L. H. (2020). Associations between Antiretroviral Drugs on Depressive Symptomatology in Homogenous Subgroups of Women with HIV. J Neuroimmune Pharmacol. 16(1):181-194.

Walmsley, S., Baumgarten, A., Berenguer, J., Felizarta, F., Florence, E., Khuong-Josses, M. A., Kilby, J. M., Lutz, T., Podzamczer, D., Portilla, J., Roth, N., Wong, D., Granier, C., Wynne, B., \& Pappa, K. (2015). Brief Report: Dolutegravir Plus Abacavir/Lamivudine for the Treatment of HIV-1 Infection in Antiretroviral Therapy-Naive Patients: Week 96 and Week 144 Results From the SINGLE Randomized Clinical Trial. Journal of acquired immune deficiency syndromes (1999), 70(5), 515-519.

WHO publishes new Consolidated HIV guidelines for prevention, treatment, service delivery \& monitoring - news release, https://www.who.int/publications/i/item/9789240031593 